\title{
Broadband Physics-Based Modeling of Microwave Passive Devices through Frequency Mapping
}

\author{
John W. Bandler, ${ }^{1,2}$ Mostafa A. Ismail,, ${ }^{1}$ José E. Rayas-Sánchez ${ }^{1}$ \\ ${ }^{1}$ Simulation Optimization Systems Research Laboratory, Department of Electrical and Computer \\ Engineering, McMaster University, Hamilton, Ontario, Canada L8S 4K1 \\ ${ }^{2}$ Bandler Corporation, P.O. Box 8083, Dundas, Ontario, Canada L9H 5E7
}

Received 5 October 2000; accepted 14 December 2000

\begin{abstract}
We present a new computer-aided modeling methodology to develop physicsbased models for passive components. We coherently integrate full-wave electromagnetic simulators, artificial neural networks, multivariable rational functions, dimensional analysis, and frequency mapping to establish broadband models. We consider both frequency-independent and frequency-dependent empirical models. Frequency mapping is used to develop the frequency-dependent empirical models. Useful properties of the frequency mapping are presented and utilized in the modeling process. We also consider the transformation from frequency-dependent models into frequency-independent ones in addition to the passivity of the frequency-dependent empirical model. We illustrate the modeling process through various examples, including a microstrip right angle bend, a microstrip via, a microstrip double-step (to be used as a basic element of constructing a model for nonuniform or tapered microstrip transmission lines), and a coplanar waveguide step junction. (c) 2001 John Wiley \& Sons, Inc. Int J RF and Microwave CAE 11: 156-170, 2001.
\end{abstract}

Keywords: empirical models; modeling; passive devices; frequency mapping; rational functions; neural networks

\section{INTRODUCTION}

We present a new computer-aided modeling methodology to develop physics-based empirical models for microwave passive components. We integrate in a coherent way electromagnetic (EM) simulators, artificial neural networks $[1,2]$, multivariable rational functions [3], dimensional analysis $[4,5]$, and frequency mapping $[6,7]$ to establish models valid over broad frequency ranges. We consider frequency-independent empirical models (FIEM) and frequency-dependent empirical mod-

Correspondence to: John W. Bandler; e-mail: bandler@ mcmaster.ca.

Contract grant sponsor: Natural Sciences and Engineering Research Council of Canada (NSERC).

Contract grant number: OGP0007239.

Contract grant number: STP0201832.

Contract grant sponsor: Micronet Network of Centres of Excellence. els (FDEM). In the FDEM we use the frequency mapping approach [6, 7] which implicitly introduces frequency dependency into the model elements. We also exploit the odd property of the frequency mapping, that is, the transformed frequency must be an odd function of the original frequency. Artificial neural networks or rational functions are used to approximate these elements as well as the frequency mapping. Rational functions enable us to transform a simple FDEM to an equivalent FIEM. This transformation can be expedited by impedance synthesis [8] as we will see in the examples. The passivity of the FDEMs is also considered. Dimensional analysis [4, 5] determines the functionality of the model elements and the frequency mapping on the components' geometrical and physical parameters. It also reduces the amount of training data required in the approximation process. The data required 
to develop the empirical models are obtained by accurate but time-intensive full-wave EM simulators (referred to in the space mapping literature as "fine" models [6]). We illustrate the process through various examples, including a microstrip right angle bend, a microstrip via, a microstrip double-step (to be used as a basic element of constructing a model for nonuniform or tapered microstrip transmission lines), and a coplanar waveguide (CPW) step junction.

Equivalent circuits can be obtained from the literature or can be visualized by microwave engineers through their understanding of and expertise with microwave components. We believe that, though simple, they have advantages over blackbox modeling of microwave components since they embody physical characteristics (at least at low frequencies) of the actual components. A shortcoming is that those equivalent circuits may not be accurate at high frequencies due to dispersion. We address dispersive effects by introducing the frequency dependency into the elements of the equivalent circuits.

\section{FREQUENCY-INDEPENDENT EMPIRICAL MODELS}

Consider a microwave component modeled by a fine model (typically a full-wave EM simulator) and a circuit model (empirical model). We assume that the topology of the equivalent circuit is known but the empirical formulas of their elements are to be determined. This concept is shown in Figure 1. The vector $\boldsymbol{x}_{f}$ is an $n$-dimensional vector representing the parameters of the microwave component and $\boldsymbol{\omega}$ is the frequency. The vectors $\boldsymbol{R}_{f}$ and $\boldsymbol{R}_{c}$ represent the fine and circuit model responses, respectively. The development of the FIEM is shown in Figure 2. The vector $y$ is an $l$-dimensional vector representing the empirical formulas of the elements of the circuit model. Applying dimensional analysis $[4,5]$ the vector $y$ becomes a function of an $n_{r}$-dimensional vector $\boldsymbol{x}_{r}\left(n_{r}<n\right)$, which we call the reduced input pa-

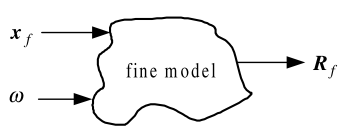

(a)

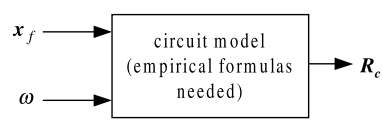

(b)
Figure 1. The fine model (a) and the circuit model (b).

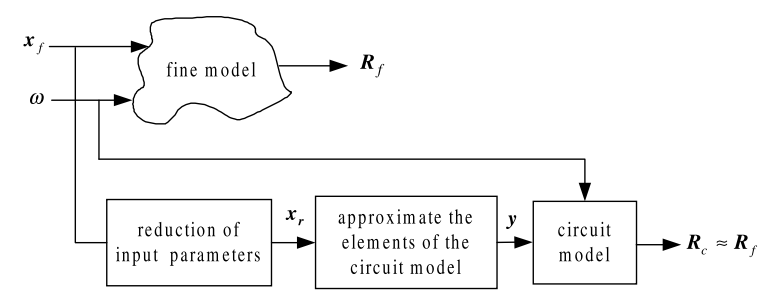

Figure 2. The development of the frequency-independent empirical models.

rameter vector (we will show in the examples how to construct this vector). We approximate $y$ through artificial neural networks $[1,2]$ or multivariable rational functions [3] in a certain region of parameters and frequency as

$$
y \approx Q\left(x_{r}, w\right)
$$

where $\boldsymbol{w}$ is a set of unknown parameters. The set $\boldsymbol{w}$ is evaluated by solving the optimization problem

$$
\min _{\boldsymbol{w}}\left\|\left[\begin{array}{lllllllll}
\boldsymbol{e}_{11}^{T} & \cdots & \boldsymbol{e}_{1 M}^{T} \boldsymbol{e}_{21}^{T} & \cdots & \boldsymbol{e}_{2 M}^{T} & \cdots & \boldsymbol{e}_{N 1}^{T} & \cdots & \boldsymbol{e}_{N M}^{T}
\end{array}\right]^{T}\right\|
$$

where \|\| is a suitable norm, $N$ is the total number of training points, $M$ is the number of frequency points per frequency sweep, and $\boldsymbol{e}_{i j}$ is an error vector given by

$$
\boldsymbol{e}_{i j}=\boldsymbol{R}_{f}\left(\boldsymbol{x}_{f_{i}}, \omega_{j}\right)-\boldsymbol{R}_{c}\left(\boldsymbol{Q}\left(\boldsymbol{x}_{r i}, \boldsymbol{w}\right), \omega_{j}\right)
$$

The optimization problem in (2) is solved by the Huber optimizer implemented in OSA90 [13]. The training points are selected according to the central composite design [9], and more training points are added if necessary.

\section{FREQUENCY-DEPENDENT EMPIRICAL MODELS}

Two approaches can be used to introduce frequency dependency to the elements of the FDEM. One approach is to introduce the frequency dependency directly to the vector $y$ (Figure 3 ). The second approach exploits the frequency mapping (transformation) concept [6,7], where we simulate the circuit model at a different frequency from the fine model. We call this frequency the circuit model frequency $\omega_{c}$. Frequency mappings (transformations) have roots in classical filter design, 


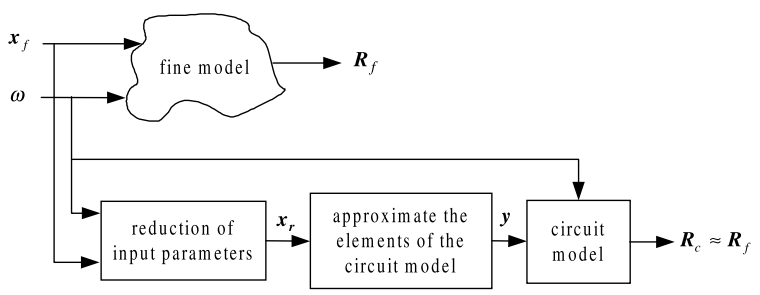

Figure 3. The development of the frequency-dependent empirical models with circuit model elements explicitly a function of frequency.

for example, low-pass to band-pass or high-pass transformations [10]. The development of the FDEM using this approach is shown in Figure 4. The dependency of $\omega_{c}$ on $\omega$ and the physical parameters are determined by applying dimensional analysis. Artificial neural networks or multivariable rational functions are used to approximate $y$ and $\omega_{c}$

$$
\begin{aligned}
y & \approx Q\left(x_{r}, w_{1}\right) \\
\omega_{c} & \approx \Omega\left(x_{r}, \omega, w_{2}\right)
\end{aligned}
$$

where $\boldsymbol{w}_{1}$ and $\boldsymbol{w}_{2}$ are unknown parameters. These parameters are evaluated by solving the optimization problem in (2) with the error vector $\boldsymbol{e}_{i j}$ given by

$\boldsymbol{e}_{i j}=\boldsymbol{R}_{f}\left(\boldsymbol{x}_{f_{i}}, \omega_{j}\right)-\boldsymbol{R}_{c}\left(\boldsymbol{Q}\left(\boldsymbol{x}_{r i}, \boldsymbol{w}_{1}\right), \Omega\left(\boldsymbol{x}_{r i}, \omega_{j}, \boldsymbol{w}_{2}\right)\right)$

\section{Properties of the Frequency Mapping}

Simulating the circuit model at a different frequency from that of the fine model is an implicit way of introducing frequency dependency to the elements of the circuit model. For example, if the device is lossless, the circuit model contains only lossless lumped-elements (inductors and capaci-

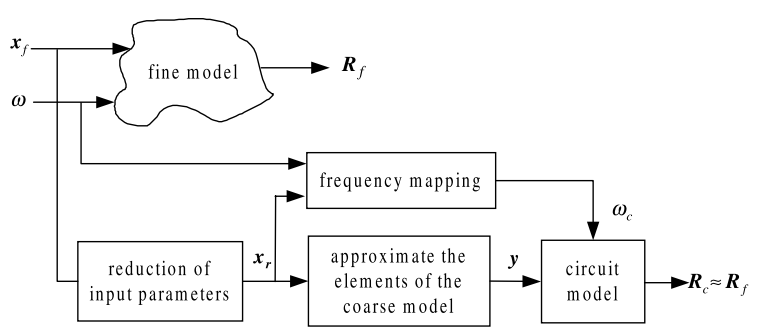

Figure 4. The development of the frequency-dependent empirical models with the circuit model elements implicitly a function of frequency through frequency mapping. tors). In this case, a FDEM simulated at $\omega_{c}$ and with a circuit element vector $\boldsymbol{y}$ is equivalent to a FDEM simulated at $\omega$ and with a circuit element vector $\boldsymbol{y}_{1}$ given by

$$
\boldsymbol{y}_{1}=\left(\omega_{c} / \omega\right) \boldsymbol{y}
$$

This can be proved as follows. For any inductor $L$ and capacitor $C$ (simulated at frequency $\omega_{c}$ ) in $y$ we have

$$
\begin{aligned}
& Z_{L}=j \omega\left(L \omega_{c} / \omega\right) \\
& Y_{C}=j \omega\left(C \omega_{c} / \omega\right)
\end{aligned}
$$

Therefore, the circuit elements vector $\boldsymbol{y}_{1}$ (simulated at frequency $\omega$ ) is related to the vector $\boldsymbol{y}$ by (6). Furthermore, the frequency $\omega_{c}$ should be an odd function of $\omega$. This results from the even and odd properties [10] of an arbitrary frequency-dependent impedance $Z(\omega)$, where the real (imaginary) part should be an even (odd) function of frequency. For example, if an inductor $L$ is simulated at frequency $\omega_{c}$ the equivalent impedance $Z_{L}=j \omega_{c} L$ is purely imaginary; hence $Z_{L}$ and consequently $\omega_{c}$ should be odd functions of $\omega$. The odd property is also preserved when using the frequency mapping to transform a low-pass filter into a high- or a band-pass filter [10]. We use this property in conjunction with dimensional analysis to further reduce the number of parameters of the artificial neural network or the multivariable rational function approximating $\omega_{c}$.

\section{Transformation of FDEMs into FIEMs}

The advantage of using a multivariable rational function to approximate the frequency mapping is that we can transform the FDEM into an equivalent FIEM. This transformation involves one-port impedance synthesis, which states that the impedance we want to realize should be a positive real rational function [8]. For example, the impedances associated with an inductor $L$ and a capacitor $C$ (simulated at $\omega_{c}$ ) in the circuit elements vector $\boldsymbol{y}$ are $Z_{L}=j \omega_{c} L$ and $Z_{C}=$ $1 /\left(j \omega_{c} C\right)$, respectively. Those impedances can be realized using any of the one-port impedance synthesis techniques such as the first Foster realization or second Foster realization or ladder 
realization [8]. In the examples presented here, we notice that the frequency $\omega_{c}$ takes the form

$$
\omega_{c}=\omega \frac{f_{1}-\omega^{2} f_{2}}{f_{3}-\omega^{2} f_{4}}
$$

where $f_{1}, f_{2}, f_{3}$, and $f_{4}$ are functions of the device physical parameters. Therefore, the impedances associated with an inductor $L$ and a capacitor $C$ in the circuit elements vector $\boldsymbol{y}$ are given by

$$
\begin{aligned}
& Z_{L}=j \omega L \frac{f_{1}-\omega^{2} f_{2}}{f_{3}-\omega^{2} f_{4}}, \\
& Z_{C}=\frac{1}{j \omega C} \frac{f_{3}-\omega^{2} f_{4}}{f_{1}-\omega^{2} f_{2}}
\end{aligned}
$$

We believe that (8) may be useful for other devices such as microstrip mitered bends, microstrip step junctions, etc.

\section{Passivity of the FDEMs}

The FDEM of a microwave component is passive if the equivalent impedance of each element (inductor or capacitor) of the circuit model is realizable. That is, the equivalent impedances given by (9a) and (9b) are realizable. An impedance $Z(s)$, where $s=j \omega$, is realizable if and only if it is a positive real function of $s$; i.e., $Z(s)$ is a real rational function of $s$ and $\operatorname{Re}(Z(s)) \geq 0$ if $\operatorname{Re}(s)$ $\geq 0$ [8]. For an LC impedance this implies that all poles of $Z(s)$ are simple and lie on the $j \omega$ axis and have positive real residues. Applying these conditions to the impedances in (9a) and (9b) and performing some algebraic manipulations we get the passivity conditions of the FDEMs (see Appendix A). A FDEM is passive if the circuit elements (inductors and capacitors) are positive and the parameters of the frequency mapping in
(8) satisfy

$$
\begin{gathered}
f_{i}>0, i=1, \ldots, 4 \\
\left|\begin{array}{ll}
f_{1} & f_{2} \\
f_{3} & f_{4}
\end{array}\right|>0
\end{gathered}
$$

Therefore to ensure the passivity of the FDEMs (10a) and (10b) should be included as constraints to the optimization problem in (2).

\section{MULTIVARIABLE RATIONAL FUNCTIONS}

Multivariable rational functions (MRFs) [3] are used in most of the modeling examples developed here. A multivariable rational function is the quotient of two polynomials,

$$
\begin{aligned}
& f(\boldsymbol{x}, \boldsymbol{a}, \boldsymbol{b}) \\
& \quad=\frac{a_{0}+\sum_{i=1}^{n} a_{i} x_{i}+\sum_{i=1}^{n} \sum_{j \geq i}^{n} a_{i j} x_{i} x_{j}+\cdots}{1+\sum_{i=1}^{n} b_{i} x_{i}+\sum_{i=1}^{n} \sum_{j \geq i}^{n} b_{i j} x_{i} x_{j}+\cdots}
\end{aligned}
$$

where $\boldsymbol{x}=\left[\begin{array}{lll}x_{1} x_{2} & \cdots & x_{n}\end{array}\right]^{T}$ is the input vector and $\boldsymbol{a}$ and $\boldsymbol{b}$ are two vectors containing the unknown $a$ 's and $b$ 's, respectively. The polynomials in the numerator and the denominator are of finite order $p$ and $q$, respectively. The rational function in (11) is fully characterized by the number of input variables $n$, the numerator order $p$, and the denominator order $q$; hence, we refer to it as $\mathrm{MRF}_{n, p, q}$. The number of unknown parameters in $\boldsymbol{a}$ and $\boldsymbol{b}$ can be reduced if some of the input variables are restricted to a certain order less than $p$ or $q$. For example, a $\mathrm{MRF}_{2,3,2}$ with the order of the input variable $x_{1}$ restricted to 1 is given by

$$
f(\boldsymbol{x}, \boldsymbol{a}, \boldsymbol{b})=\frac{a_{0}+a_{1} x_{1}+a_{2} x_{2}+a_{12} x_{1} x_{2}+a_{22} x_{2}^{2}+a_{122} x_{1} x_{2}^{2}+a_{222} x_{2}^{3}}{1+b_{1} x_{1}+b_{2} x_{2}+b_{12} x_{1} x_{2}+b_{22} x_{2}^{2}}
$$

which has 11 unknown parameters. On the other hand, the full $\mathrm{MRF}_{2,3,2}$ has 15 unknown parameters. The unknown parameters in $\boldsymbol{a}$ and $\boldsymbol{b}$ can be computed by two methods. First, if the values of the function $f$ in (11) are explicitly available we can evaluate $\boldsymbol{a}$ and $\boldsymbol{b}$ by solving a system of linear equations. This is done by applying cross-multiplication to both sides of (11) and rearranging the terms to get a system of linear equations in the elements of $\boldsymbol{a}$ and $\boldsymbol{b}$. This system of linear equations can be solved by the method of least-squares or a recursive least-squares algorithm [3]. Second, 
if values of $f$ are not directly available we evaluate $\boldsymbol{a}$ and $\boldsymbol{b}$ by solving a suitable optimization problem [in our case the optimization problem in (2)]. The second method is adopted in this work since we evaluate the elements of the empirical model (inductors, capacitors, and the frequency $\omega_{c}$ ) and the scattering parameters are the only available information supplied by the EM simulators.

\section{MODELING EXAMPLES}

To display the results in a compact way we define the error in the scattering parameter $S_{i j}$ as the modulus of the difference between the scattering parameter $S_{i j}^{f}$ computed by the fine model and the scattering parameter $S_{i j}^{c}$ computed by the circuit model

$$
\text { error in } S_{i j}=\left|S_{i j}^{f}-S_{i j}^{c}\right|=\sqrt{\left(\operatorname{Re}\left[S_{i j}^{f}\right]-\operatorname{Re}\left[S_{i j}^{c}\right]\right)^{2}+\left(\operatorname{Im}\left[S_{i j}^{f}\right]-\operatorname{Im}\left[S_{i j}^{c}\right]\right)^{2}}
$$

where $i=1,2, \ldots, P$ and $j=1,2, \ldots, P$ ( $P$ is the number of ports of the microwave device). We also define the percentage error in $S_{i j}$ by

$$
\% \text { error in } S_{i j}=\left|\frac{S_{i j}^{f}-S_{i j}^{c}}{S_{i j}^{f}}\right| \times 100
$$

We will use percentage error in $S_{i j}$ to display the results whenever $\left|S_{i j}^{f}\right|$ is not zero.

\section{Microstrip Right Angle Bend}

Here, we develop a frequency-independent and frequency-dependent empirical model for the microstrip right angle bend in Figure 5(a). The fine model is analyzed by Sonnet's $\mathbf{e m}$ [11] and the circuit model is the LC circuit [12] in Figure 5(b). The vector of input parameters $\boldsymbol{x}_{f}=\left[\begin{array}{lll}W & H & \varepsilon_{r}\end{array}\right]^{T}$ and the vector of the circuit elements is $y=$ $[L / H C / H]^{T}$. Applying dimensional analysis $[4$, 5], we can show that the elements of $y$ are given by

$$
\begin{aligned}
& L / H=\mu_{0} f(W / H) \\
& C / H=\varepsilon_{0} f\left(W / H, \varepsilon_{r}\right)
\end{aligned}
$$

Therefore, $\boldsymbol{y}$ is a function of $\boldsymbol{x}_{r}=\left[\begin{array}{lll}W / H & \varepsilon_{r}\end{array}\right]^{T}$. We first develop a FIEM in the frequency range $[1,11] \mathrm{GHz}$. The region of interest is $0.2<W / H$ $<6$ and $2<\varepsilon_{r}<11$. The substrate height $H$ is chosen in the range $[5,30]$ mil. We use a threelayer perceptron artificial neural network (ANN) (with hyperbolic-tangent as nonlinear activation function) to approximate $\boldsymbol{y}$. Two hidden neurons are used for $L / H$ and three hidden neurons for $C / H$. The training points are chosen according to the central composite design [9] in addition to 4 more points as shown in Figure 6 (total 13 training points) where $\hat{x}_{1}$ and $\hat{x}_{2}$ are the scaled input

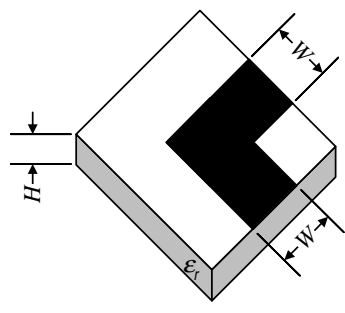

(a)

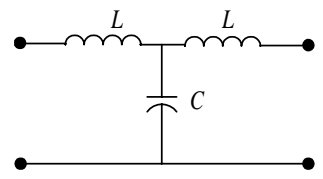

(b)
Figure 5. The microstrip right angle bend: (a) the fine model; (b) the circuit model.

variables corresponding to $W / H$ and $\varepsilon_{r}$, respectively. The vector $\boldsymbol{y}$ is also approximated by multivariable rational functions. The inductance per unit length $L / H$ is approximated by a rational function $\mathrm{MRF}_{1,2,2}$ and the capacitance per unit length $C / H$ is approximated by a rational function $\mathrm{MRF}_{2,3,0}$ with the order of $W / H$ restricted to one (this gives better generalization performance than if we did not restrict the order of $W / H)$. The parameters of the ANNs and the MRFs are obtained by the Huber optimizer in

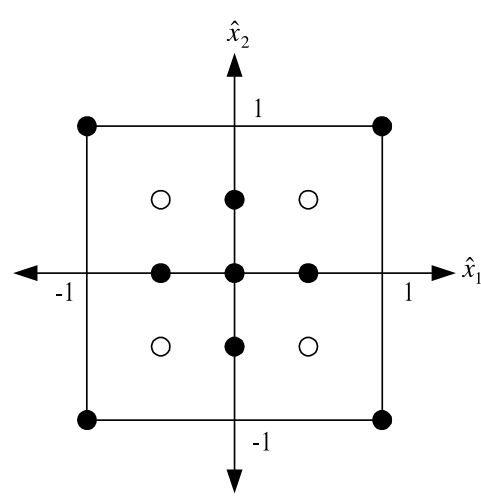

Figure 6. The training points for the microstrip right angle bend. 


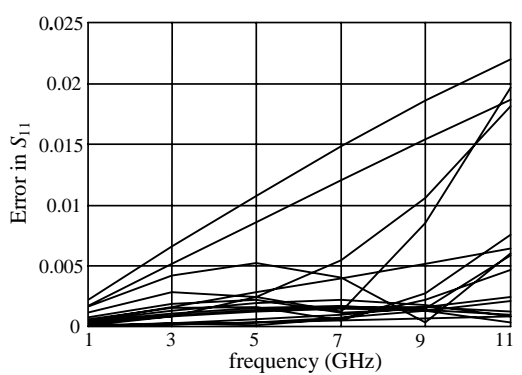

(a)

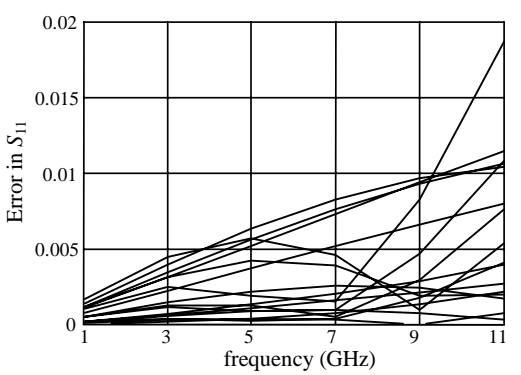

(b)

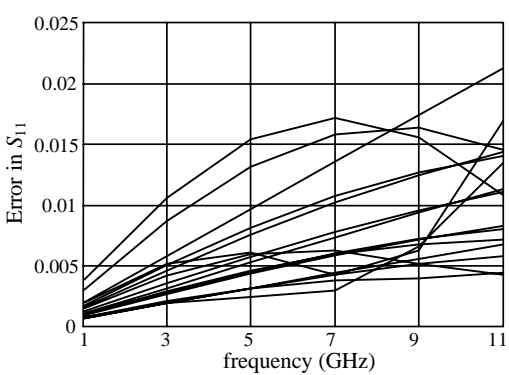

(c)

Figure 7. The error in $S_{11}$ of the microstrip right angle bend with respect to $\boldsymbol{e m}$ at the test points: (a) the FIEM developed by ANNs; (b) the FIEM developed by MRFs; (c) the FIEM developed by the empirical model in [14].

OSA90/hope [13]. Figures 7(a) and 7(b) show the error in the scattering parameter $S_{11}$ at 16 test points in the region of interest for the FIEM developed by ANN and MRF, respectively. Figure 7(c) shows the corresponding error due to the Jansen model [14] at the same test points. We see that the three models are comparable.

The results obtained by the FIEM (developed by either ANNs or MRFs) and by the Jansen empirical model [14] over a broad frequency range are shown in Figures 8(a), 8(b) and 8(c), respectively. It is clear that neither the FIEM nor the empirical model in [14] is accurate at high frequencies. Therefore, we develop a FDEM (see Figure 4), where $\omega_{c}$ is a function of $\omega$ and the other parameters. Applying dimensional analysis (see Appendix B) and using the odd property of $\omega_{c}$ we get

$$
\omega_{c}=\omega \gamma\left(x_{r},(\omega H / c)^{2}\right)
$$

where $c$ is the speed of light and $\gamma$ is an unknown function to be approximated. We use multivariable rational functions to approximate $\boldsymbol{y}$ as well as $\omega_{\mathrm{c}} \cdot \mathrm{A} \mathrm{MRF}_{3,2,2}$ with the order of $(\omega H / c)^{2}$ restricted to one is used to approximate $\omega_{c}$. The number of training points used to develop the FDEM is the same as that used to develop the FIEM. Figures 9(a) and 9(b) show the errors in the scattering parameters $S_{11}$ and $S_{21}$ at 16 test points in the region of interest for the FDEM. Figure 10 compares the results obtained by the FDEM and those from Sonnet's em [11]. The empirical expressions for $\boldsymbol{y}$ and $\omega_{c}$ are given in Table I.

We transform the FDEM into an equivalent FIEM as follows. The frequency $\omega_{c}$ is given by (8) and, hence, the impedances associated with $L$ and $C$ are given by (9a) and (9b), respectively. These impedances are realized by the first Foster realization [8]. The equivalent FIEM is shown in Figure 11(b), where all elements are frequency

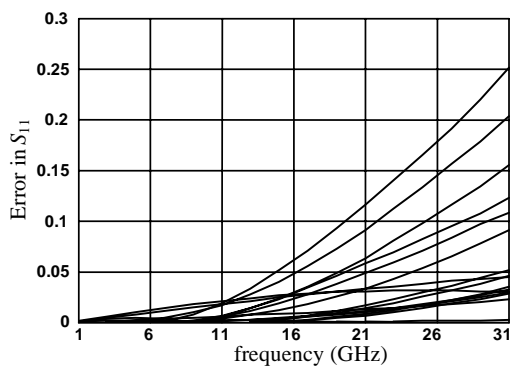

(a)

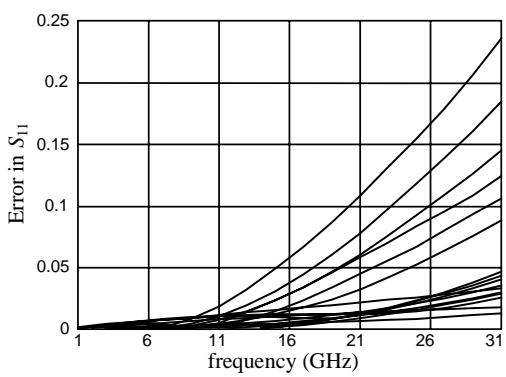

(b)

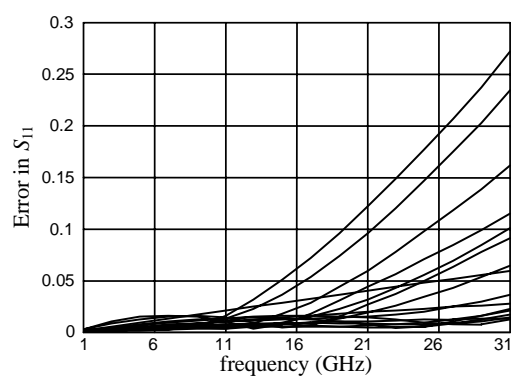

(c)

Figure 8. The error in $S_{11}$ of the microstrip right angle bend with respect to $\mathbf{e m}$ over a broad frequency range: (a) the FIEM developed by ANNs; (b) the FIEM developed by MRFs; (c) the FIEM developed by the empirical model in [14]. 


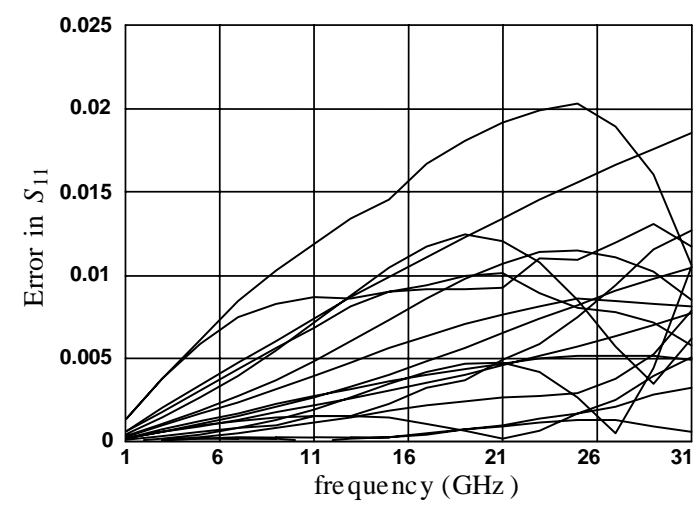

(a)

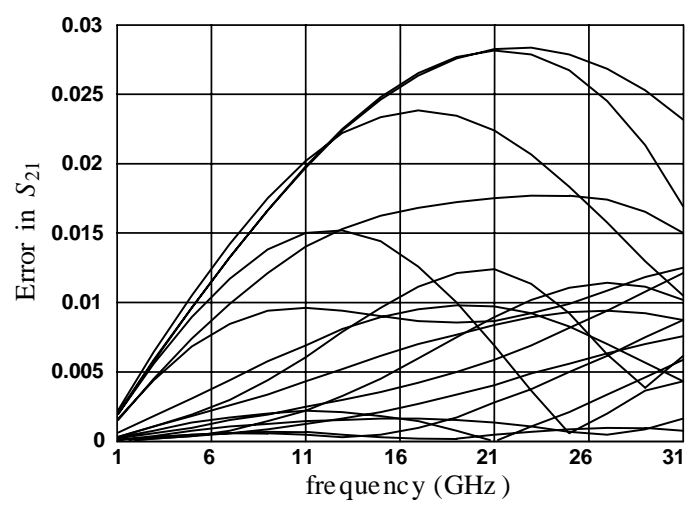

(b)

Figure 9. The error of the FDEM of the microstrip right angle bend (developed by MRFs) with respect to $\boldsymbol{e m}$ at the test points: (a) in $S_{11}$; (b) in $S_{21}$.

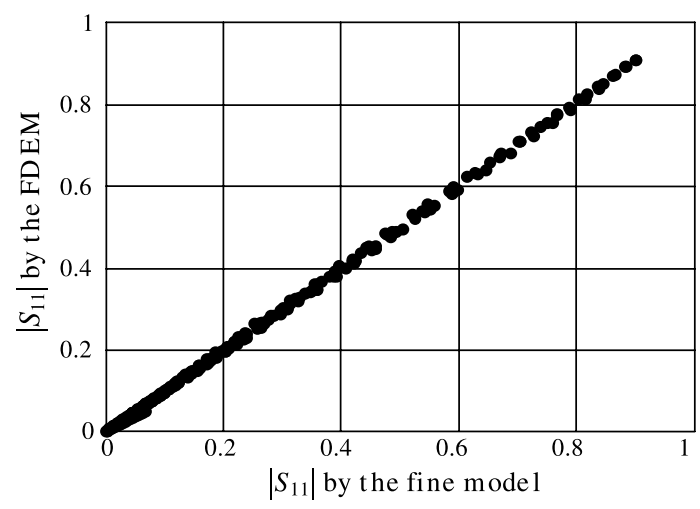

(a)

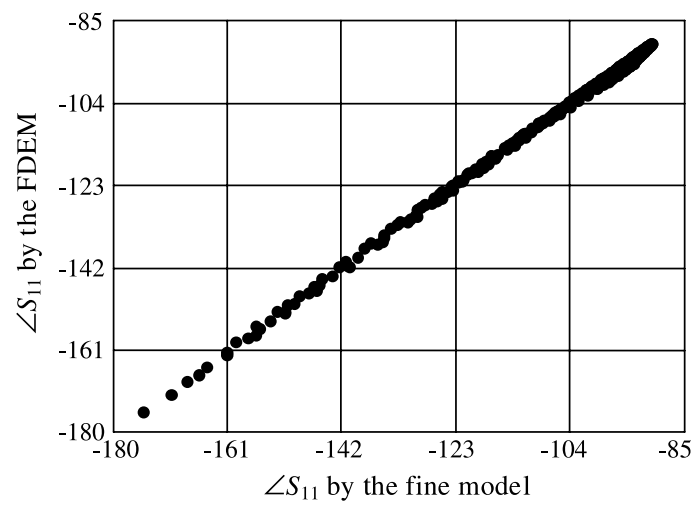

(b)

Figure 10. Comparison between the responses obtained by the FDEM of the microstrip right angle bend and those obtained by $\boldsymbol{e m}$ at the test points: (a) magnitude of $S_{11}$; (b) phase of $S_{11}$ in degrees.

TABLE I. Expressions of the Elements of the FDEM of the Microstrip Right Angle Bend

\begin{tabular}{lc}
\hline Element & Expression \\
\hline$L / H(\mathrm{nH} / \mathrm{mil})$ & $0.03192 \frac{-0.09-0.018 x_{1}+0.3 x_{1}^{2}}{1+2.853 x_{1}^{2}}$ \\
$C / H(\mathrm{pF} / \mathrm{mil})$ & $0.000225\left(-0.46+0.162 x_{1}-0.014 x_{2}+0.275 x_{1}^{2}+2.855 x_{1} x_{2}+0.262 x_{1}^{2} x_{2}\right)$ \\
$\omega_{c} / \omega$ & $\frac{f_{1}\left(x_{1}, x_{2}, x_{3}\right)}{f_{2}\left(x_{1}, x_{2}, x_{3}\right)}$ \\
& \\
& $f_{1}\left(x_{1}, x_{2}, x_{3}\right)=0.759-0.0192 x_{1}-0.0179 x_{2}+0.0187 x_{3}+0.0738 x_{1}^{2}+0.0026 x_{1} x_{2}$ \\
& $-0.1405 x_{1} x_{3}+0.0079 x_{2} x_{3}+0.0018 x_{1}^{3}-0.0071 x_{1}^{2} x_{2}+0.1188 x_{1}^{2} x_{3}$ \\
& $+0.0017 x_{1} x_{2}^{2}+0.0419 x_{1} x_{2} x_{3}-0.0022 x_{2}^{2} x_{3}$ \\
& \\
& \\
$f_{2}\left(x_{1}, x_{2}, x_{3}\right)=$ & $+0.0282 x_{1}-0.0086 x_{2}-0.0175 x_{3}+0.0051 x_{1}^{2}-0.0063 x_{1} x_{2}$ \\
& $+0.1674 x_{1} x_{3}+0.0037 x_{2}^{2}-0.0067 x_{2} x_{3}+0.0055 x_{1}^{3}-0.0028 x_{1}^{2} x_{2}$ \\
& $+0.0011 x_{1}^{2} x_{3}+0.0056 x_{1} x_{2} x_{3}-0.0012 x_{2}^{2} x_{3}$ \\
& \\
where $x_{1}=W / H, x_{2}=\varepsilon_{r}, x_{3}=1.816 e-7(\omega(\mathrm{GHz}) H(\mathrm{mil}))^{2}$
\end{tabular}




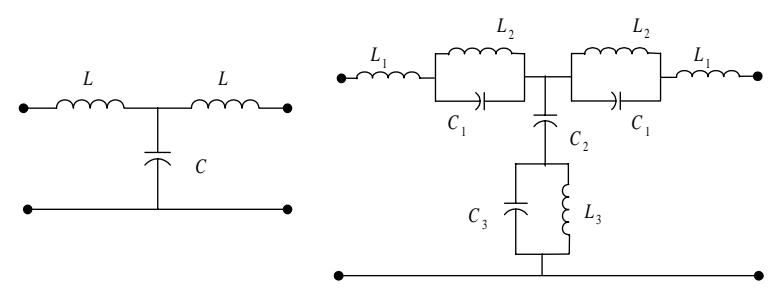

working frequency is $\omega_{c}$

(a)

working frequency is $\omega$

(b)

Figure 11. The FDEM of the microstrip right angle bend (a) and the equivalent FIEM (b).

independent and functions only of the device parameters.

\section{Microstrip Via}

Here, we consider modeling the microstrip via of Figure 12(a). The circuit model is an inductor $L$ to ground [Figure 12(b)]. The fine model is analyzed by Sonnet's em [11]. The reference plane is at the junction of the microstrip line and the square pad. The vector $\boldsymbol{x}_{f}=\left[\begin{array}{llll}W & H & W_{0} & D\end{array}\right]^{T}$, where $H$ is the substrate height (GaAs, $\varepsilon_{r}=12.9$ ). Here, $\boldsymbol{y}=[L / H]$, which is given by

$$
L / H=\mu_{0} f\left(W / H, W_{0} / W, D / W\right)
$$

hence, $\boldsymbol{x}_{r}=\left[W / H W_{0} / W D / W\right]^{T}$. A FIEM was developed in the range $[2,10] \mathrm{GHz}$. The region of interest is $1<W / H<2.2,0.2<W_{0} / W<1$ and $0.2<D / W<0.8$. We use a $\mathrm{MRF}_{3,2,2}$ to approximate $L / H$. The training points are chosen according to the central composite design [9] in addition to 8 more points (total of 23 training

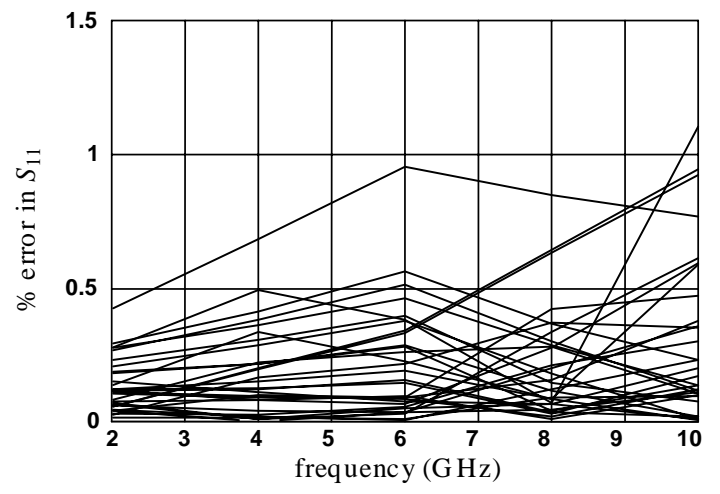

(a)

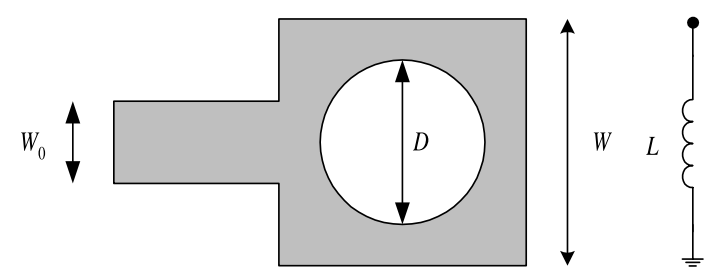

(a)

(b)

Figure 12. The microstrip via: (a) the physical structure; (b) the circuit model.

points). The parameters of the MRF are obtained by the Huber optimizer in OSA90/hope [13]. The percentage errors in the inductance $L$ and in $S_{11}$ at 30 test points are shown in Figure 13. Figure 14 compares the results obtained by the FIEM and those from Sonnet's em [11].

The results of the FIEM in the range $[2,22]$ $\mathrm{GHz}$ are shown in Figure 15. We notice large errors at high frequencies. This is because the simple inductor to ground does not take into account the effect of the pad surrounding the via hole and the step junction [15] (see Figure 12). To overcome this deficiency we develop a FDEM in the range [2, 22] $\mathrm{GHz}$. The circuit model frequency (applying dimensional analysis and using the odd property of the frequency mapping) takes the same form as in (16). We use multivariable rational functions to approximate $y$ as well as $\omega_{c}$. The number of training points used is 23. The percentage errors in $L$ and in $S_{11}$ at 30 test points are shown in Figures 16(a) and 16(b), respectively. The transformation of the FDEM into an equivalent FIEM follows the microstrip right angle bend example. The frequency $\omega_{c}$ is given by (8). The equivalent impedance of $L$ is of the form

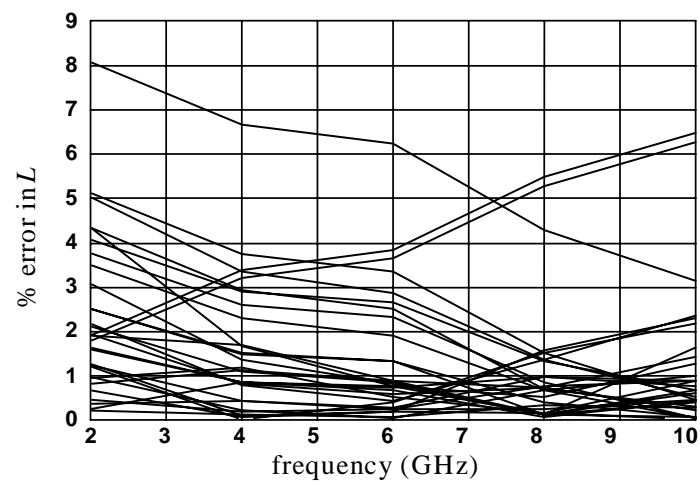

(b)

Figure 13. Percentage error of the FIEM of the microstrip via with respect to em at the test points: (a) in $S_{11}$; (b) in $L$. 


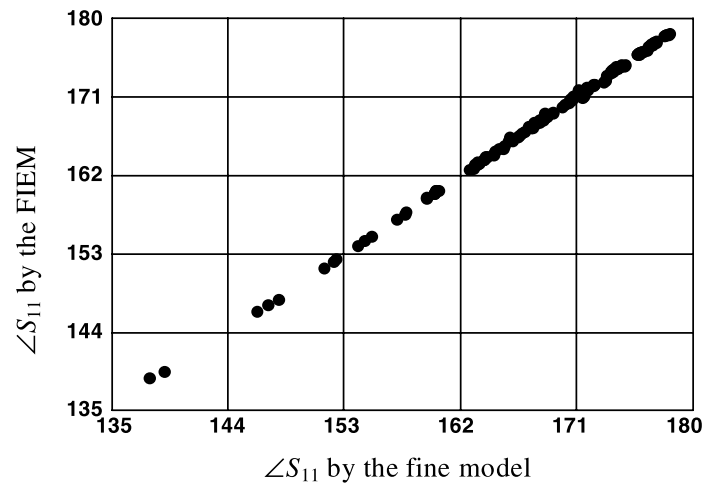

(a)

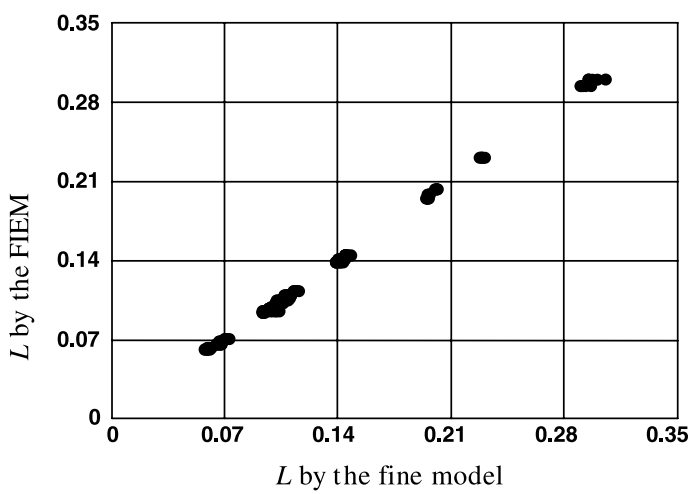

(b)

Figure 14. Comparison between the responses obtained by the FIEM of the microstrip via and those obtained by $\boldsymbol{e m}$ at the test points in the frequency range $[2,10] \mathrm{GHz}$ : (a) phase of $S_{11}$; (b) the inductance $L$.

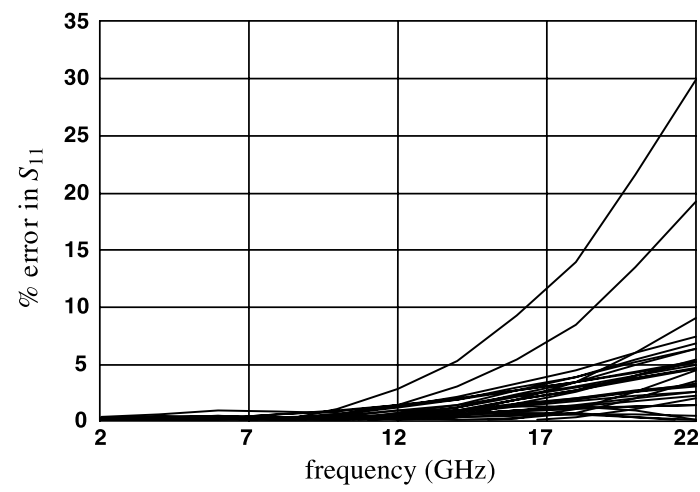

(a)

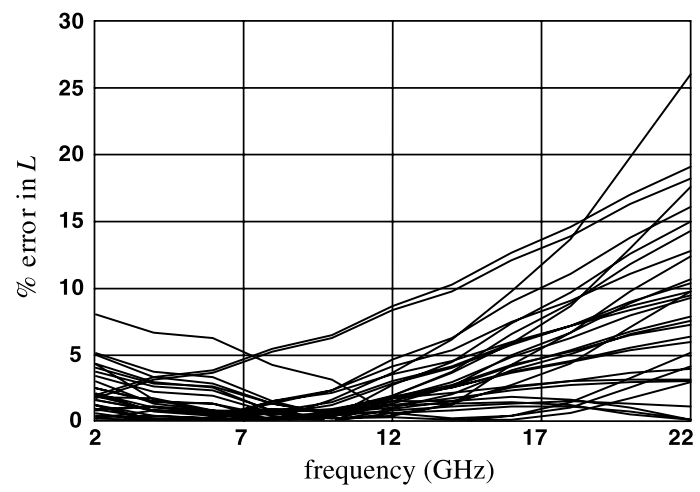

(b)

Figure 15. Comparison of the FIEM of the microstrip via with respect to em over a broad frequency range at the test points: (a) $\%$ error in $S_{11}$; (b) $\%$ error in $L$.

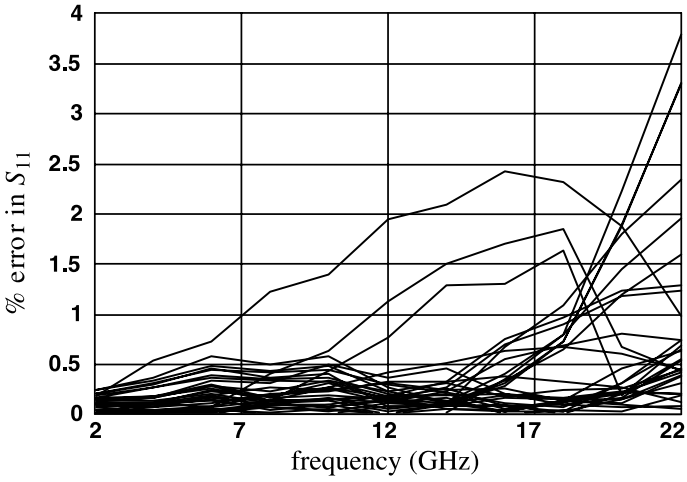

(a)

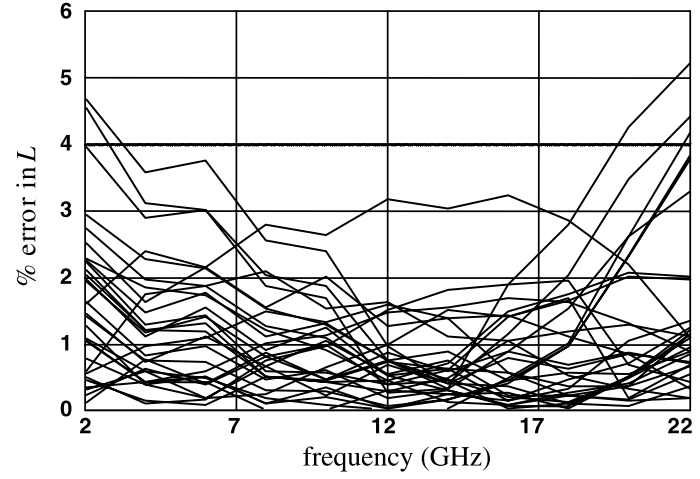

(b)

Figure 16. Comparison of the FDEM of the microstrip via with respect to $\mathrm{em}$ over a broad frequency range at the test points: (a) $\%$ error in $S_{11}$; (b) $\%$ error in $L$. 


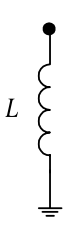

working frequency is $\omega_{c}$

(a)

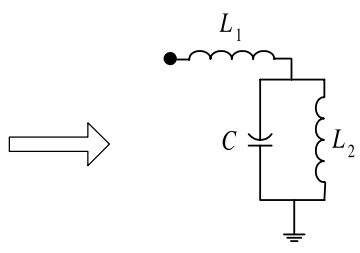

working frequency is $\omega$

(b)
Figure 17. The FDEM of the microstrip via (a) and the corresponding FIEM (b).

of (9a). The resulting FIEM is shown in Figure 17.

\section{Microstrip Double-Step}

Here, we consider broadband modeling of the microstrip double-step element in Figure 18(a). It can be used to model microstrip tapered lines or nonuniform (in width) microstrip lines. The circuit model consists of two shunt capacitances and one series inductance [Figure 18(b)]. The fine model is analyzed by Sonnet's $\boldsymbol{e m}$ [11]. The vector of fine model parameters $\boldsymbol{x}_{f}=\left[\begin{array}{lll}W_{1} & W_{2} & W_{3}\end{array}\right]^{T}$. The substrate height $H=25 \mathrm{mil}$, the relative dielectric constant $\varepsilon_{r}=9.7$ and the length $l$ [Figure 18(a)] is 5 mil. The circuit elements vector $\boldsymbol{y}=$ $\left[L_{1} / H C_{1} / H C_{2} / H\right]^{T}$. The elements of $\boldsymbol{y}$ are given by

$$
\begin{aligned}
& L_{1} / H=\mu_{0} f_{1}\left(\frac{W_{2}}{H}, \frac{W_{2}}{W_{1}}, \frac{W_{3}}{W_{2}}\right) \\
& C_{1} / H=\varepsilon_{0} f_{2}\left(\frac{W_{2}}{H}, \frac{W_{2}}{W_{1}}, \frac{W_{3}}{W_{2}}\right) \\
& C_{2} / H=\varepsilon_{0} f_{3}\left(\frac{W_{2}}{H}, \frac{W_{2}}{W_{1}}, \frac{W_{3}}{W_{2}}\right)
\end{aligned}
$$

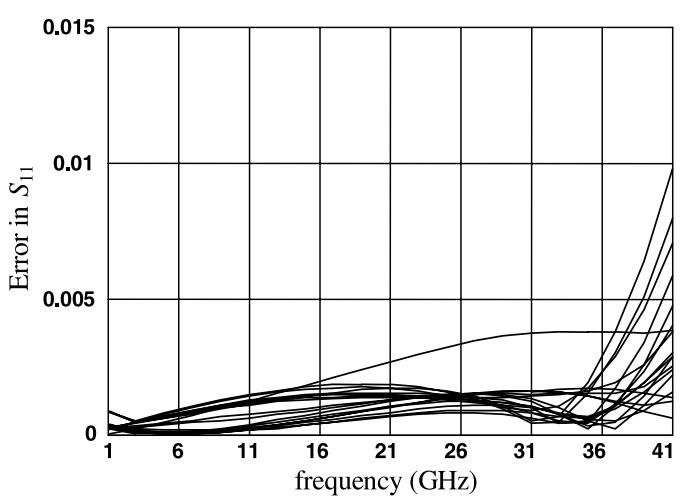

(a)

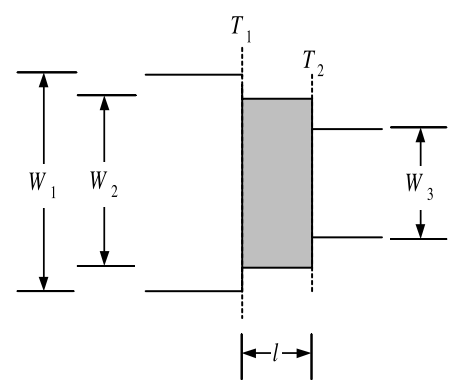

(a)

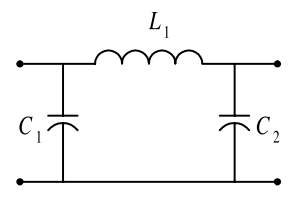

(b)
Figure 18. The microstrip double-step: (a) the physical structure where $T_{1}$ and $T_{2}$ are the reference planes; (b) the circuit model.

hence, $\boldsymbol{x}_{r}=\left[W_{2} / H W_{2} / W_{1} W_{3} / W_{2}\right]^{T}$. The circuit model frequency (applying dimensional analysis and using the odd property of the frequency mapping) takes the same form as in (16). A FDEM of the double-step element is developed in the frequency range $[1,41] \mathrm{GHz}$. The region of interest is $0.1<W_{2} / H<1,0.5<W_{2} / W_{1}<0.9$ and $0.5<W_{3} / W_{2}<0.9$. We use a $\mathrm{MRF}_{3,2,2}$ to approximate each element of the vector $y$ and a $\mathrm{MRF}_{4,2,2}$ to approximate $\omega_{c}$ with the order of $(\omega H / c)^{2}$ restricted to 1 . The number of training points is 23. The parameters of the MRFs are obtained by the Huber optimizer in OSA90/hope [13]. The errors in $S_{11}$ and $S_{21}$ of the FDEM with respect to Sonnet's $\boldsymbol{e m}$ [11] at 27 testing points in the region of interest are shown in Figure 19(a) and (b), respectively. To evaluate the FDEM of the double-step we consider an alternative model for the double-step element. This model is composed of a microstrip transmission line and two step junctions as shown in Figure 20. The empirical models for the microstrip line and the two step junctions are taken from OSA90/hope. Fig-

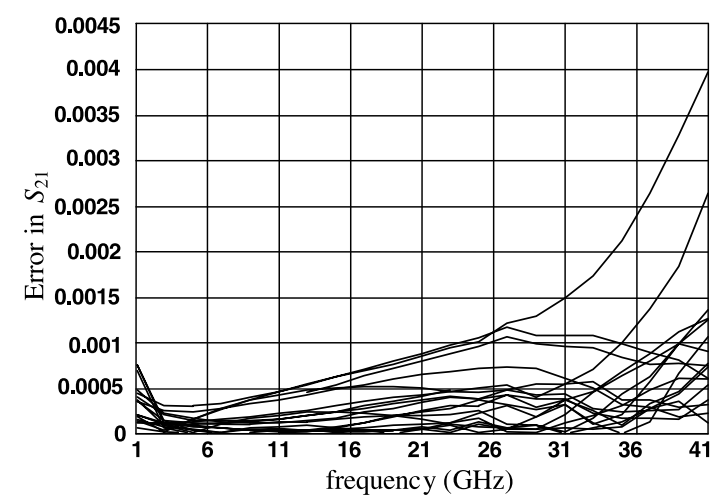

(b)

Figure 19. Comparison between the FDEM of the double-step element and $\boldsymbol{e m}$ at the test points in the region of interest: (a) error in $S_{11}$; (b) error in $S_{21}$. 


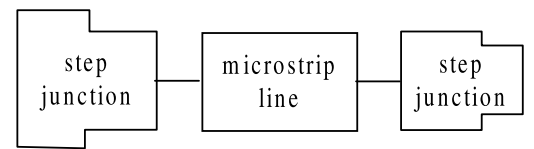

Figure 20. An alternative model for the microstrip double-step element.

ure 21(a) and (b) show the errors in $S_{11}$ and $S_{21}$ of this model with respect to Sonnet's em [11] at 27 testing points in the region of interest. It is clear from Figures 19 and 21 that the FDEM outperforms the double-step model in Figure 20.

The FDEM of the double-step element is used to model the linear tapered microstrip line in Figure 22. The parameters of the tapered line are $L=150 \mathrm{mil}, W_{\text {in }}=18 \mathrm{mil}, W_{\text {out }}=2 \mathrm{mil}, H=$ 25 mil, and $\varepsilon_{r}=9.7$. The input microstrip line has a characteristic impedance of $50 \mathrm{ohms}$ and the output line has a characteristic impedance of $100 \mathrm{ohms}$. The linear tapered microstrip line can be modeled by cascading 30 double-step elements (each of length $l=5$ mil). The ABCD matrix of the tapered line is related to the ABCD matrices of the double-step elements by

$$
\left[\begin{array}{ll}
A & B \\
C & D
\end{array}\right]=\prod_{i=1}^{30}\left[\begin{array}{ll}
A_{i} & B_{i} \\
C_{i} & D_{i}
\end{array}\right]
$$

We analyzed the tapered line by three methods: by Sonnet's $\boldsymbol{e m}$ [11] (the fine model), by cascading 30 double-step elements, where the FDEM is used to model each element and by cascading 30 elements where the alternative model of the double-step element in Figure 20 is used. Figure 23 compares the results obtained by the three methods.

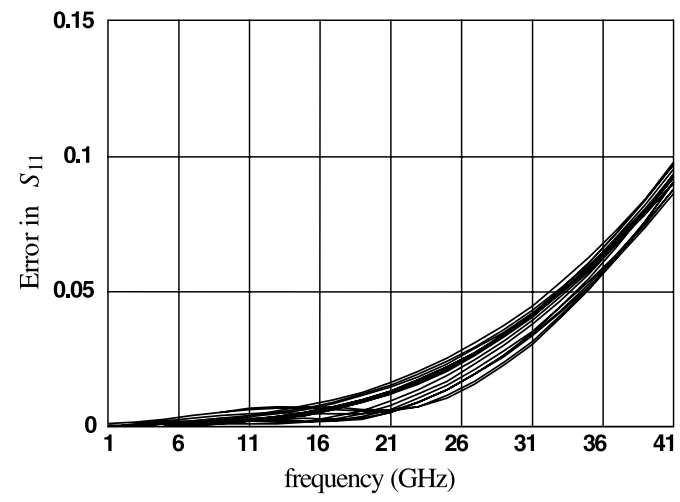

(a)

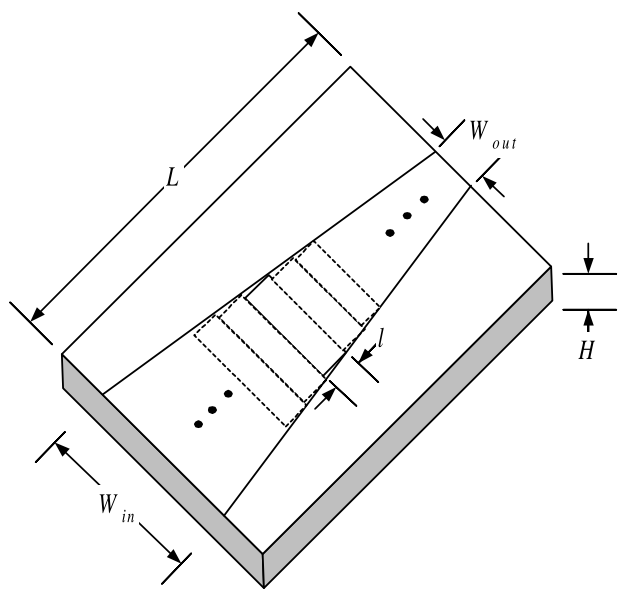

Figure 22. Linear tapered microstrip line.

\section{CPW Step Junction}

Here, we develop a FIEM for the CPW step junction in Figure 24(a). The fine model is analyzed by Sonnet's $\boldsymbol{e m}$ [11] and the circuit model is the LC circuit [12] in Figure 24. The vector of input parameters $\boldsymbol{x}_{f}=\left[\begin{array}{lll}W_{1} & W_{2} & G\end{array}\right]^{T}$ and the vector of the circuit elements is $\boldsymbol{y}=\left[L_{1} / H L_{2} / H\right.$ $\mathrm{C} / H]^{T}$, where

$$
\begin{aligned}
L_{1} / H & =\mu_{0} f_{1}\left(W_{1} / H, W_{2} / W_{1}, G / W_{1}\right) \\
L_{2} / H & =\mu_{0} f_{2}\left(W_{1} / H, W_{2} / W_{1}, G / W_{1}\right) \\
C / H & =\varepsilon_{0} f_{3}\left(W_{1} / H, W_{2} / W_{1}, G / W_{1}\right)
\end{aligned}
$$

Therefore, $\boldsymbol{y}$ is a function of $\boldsymbol{x}_{r}=\left[W_{1} / H W_{2} / W_{1}\right.$ $\left.G / W_{1}\right]^{T}$. The region of interest is $40 \mu \mathrm{m}<W_{1}<$ $120 \mu \mathrm{m}, 0.2<W_{2} / W_{1}<0.8$ and $0.2<G / W_{1}<$ 1 and the frequency range is [5, 50] GHz. The substrate height $H$ is $635 \mu \mathrm{m}$ and the relative

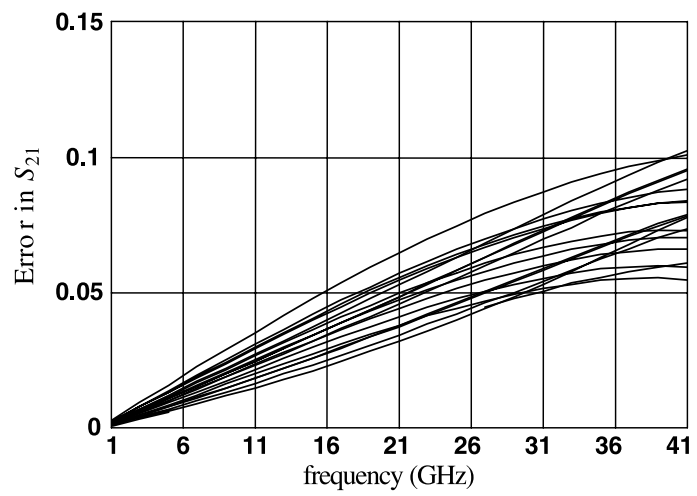

(b)

Figure 21. Comparison between the double-step model in Figure 20 and $\boldsymbol{e m}$ at the test points in the region of interest; (a) error in $S_{11}$; (b) error in $S_{21}$. 


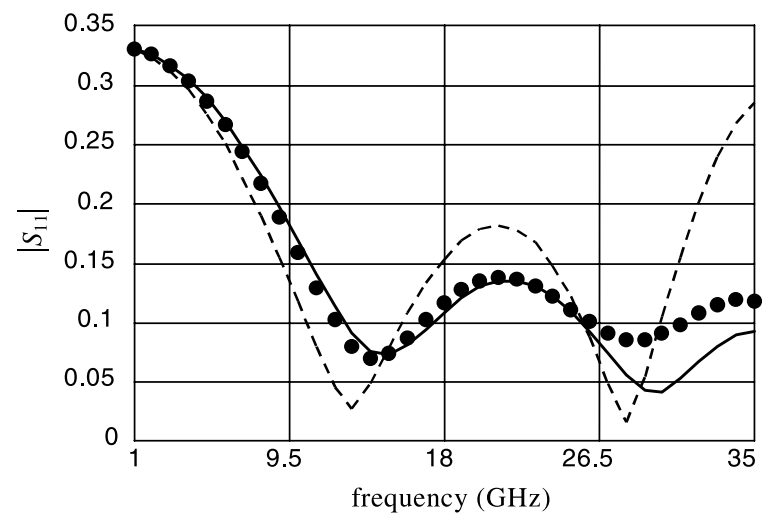

Figure 23. The response of the linear tapered microstrip line by $\boldsymbol{e m}(\bullet-\bullet)$, by the FDEM of the doublestep element (-), by the model in Figure 20 of the double-step element (---).

dielectric constant is $\varepsilon_{r}=12.9$ (GaAs). The number of training points is 23. Each element of the vector $\boldsymbol{y}$ is approximated by a rational function $\mathrm{MRF}_{3,2,2}$. The parameters of the MRFs are obtained by the Huber optimizer in OSA90/hope [13]. Figures 25(a) and 25(b) compare the results obtained by em [11] and those by the CPW step

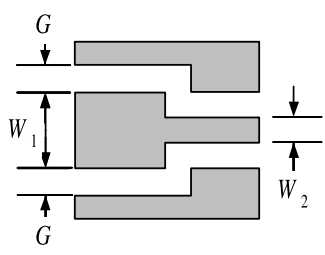

(a)

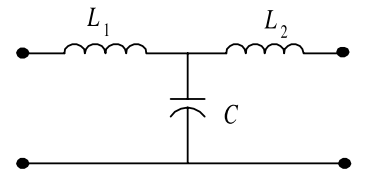

(b)
Figure 24. The CPW step junction: (a) the physical structure; (b) the circuit model.

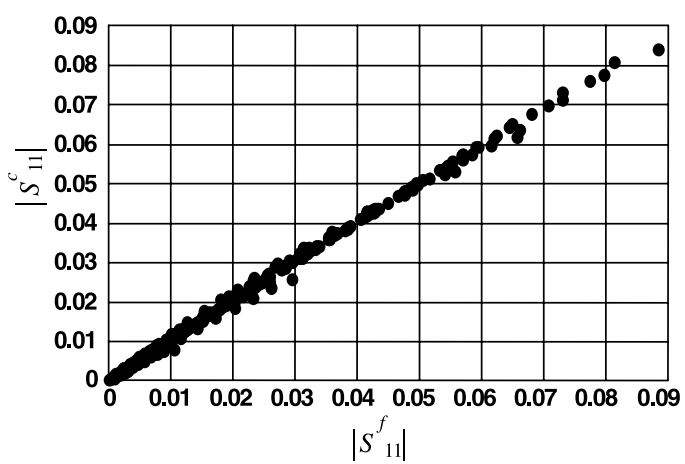

(a)

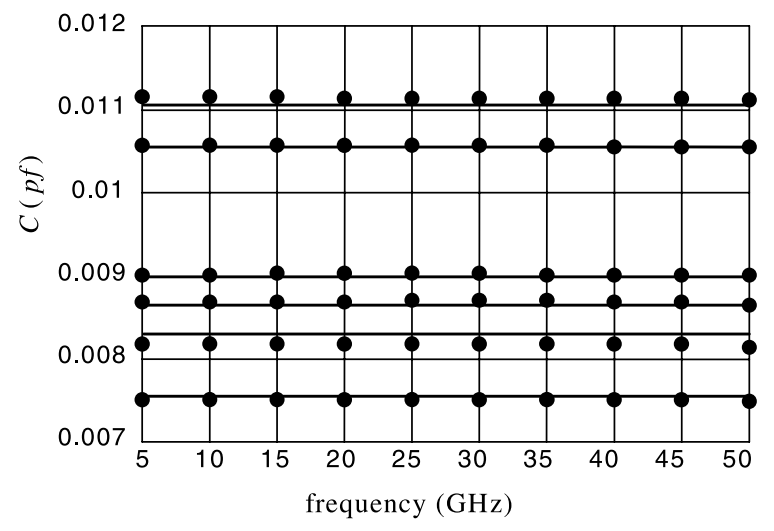

Figure 26. The capacitance of the CPW step junction: (a) extracted from the fine model $(\bullet-\bullet)$; (b) predicted by the FIEM of the CPW step junction (-).

junction FIEM at 27 test points in the region of interest. We notice that the CPW step junction FIEM gives good results in a broad frequency range from 5 to $50 \mathrm{GHz}$. Therefore, we do not need to develop a FDEM for the CPW step junction. This means that the elements of the CPW step junction empirical model are frequency independent. Figure 26 compares the capacitance $C$ extracted from the $Z$-parameters obtained by $\boldsymbol{e m}$ [11] and that predicted by the FIEM at six test points in the region of interest.

\section{CONCLUSIONS}

We present a unified computer-aided modeling methodology for developing broadband models of microwave passive components. Our approach integrates in a coherent way full-wave EM simu-

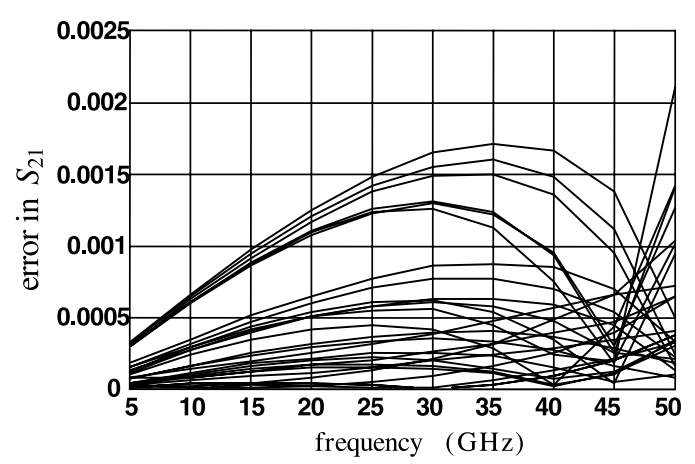

(b)

Figure 25. Comparison between the results obtained by $\boldsymbol{e m}$ and by the FIEM of the CPW step junction: (a) $\left|S_{11}\right|$ by $\boldsymbol{e m}$ versus that of the FIEM; (b) the error in $S_{21}$. 
lations, artificial neural networks, multivariable rational functions, dimensional analysis and frequency mapping. Two types of models are considered: frequency-independent and frequency-dependent empirical models. The latter can be transformed to the former if we use a rational function to approximate the frequency mapping. This is important since the frequency-independent empirical models are readily implementable in conventional circuit simulators. We also discussed the passivity condition of the frequencydependent empirical models. We applied our modeling methodology to develop broadband models for several microwave components, including a microstrip right angle bend, a microstrip via, a microstrip double-step, and a CPW step junction.

\section{APPENDIX A}

The passivity conditions for the FDEM can be proven as follows. The impedance in (9a) is written in terms of $s=j \omega$ as

$$
Z_{L}(s)=L s \frac{f_{1}+s^{2} f_{2}}{f_{3}+s^{2} f_{4}}
$$

By partial fractions we get

$$
Z_{L}(s)=L \frac{f_{2}}{f_{4}}\left(1+\frac{f_{1} / f_{2}-f_{3} / f_{4}}{f_{3} / f_{4}+s^{2}}\right)
$$

Since the poles of an LC impedance lie on the $j \omega$ axis and have positive residues, we get the following conditions

$$
\begin{aligned}
L \frac{f_{2}}{f_{4}} & >0 \\
f_{3} / f_{4} & >0 \\
f_{1} / f_{2}-f_{3} / f_{4} & >0
\end{aligned}
$$

The inductance $L$ must be positive; hence, $f_{1}, f_{2}$, $f_{3}$, and $f_{4}$ must have the same sign. If we assume that $f_{1}, f_{2}, f_{3}$, and $f_{4}$ are positive, the conditions in (A3)-(A5) are equivalent to

$$
\begin{gathered}
f_{i}>0, i=1, \ldots, 4 \\
f_{1} f_{4}-f_{2} f_{3}>0
\end{gathered}
$$

Those conditions can be also obtained by applying the same procedure to the impedance in (9b).

\section{APPENDIX B}

We apply dimensional analysis to determine the dependency of the circuit model frequency $\omega_{c}$ (in the microstrip right angle bend example) on the fine model frequency $\omega$ and the other parameters. The method of dimensional analysis is based on Buckingham's theorem [4]. This theorem states that "If an equation is dimensionally homogeneous it can be reduced to a relationship among a complete set of dimensionless products of the system variables." The dimensionless products are called pi $(\pi)$ terms $[4,5]$. For our case we assume that $\omega_{c}$ depends on $\omega$, the device parameters $W$, $H$, and $\varepsilon$, the free space permittivity $\varepsilon_{0}$ and the speed of light $c$ (we can replace $c$ with the free space permeability $\left.\mu_{0}\right)$. A dimensional product $\pi$ takes the form

$$
\pi=H^{x_{1}} W^{x_{2}} c^{x_{3}} \varepsilon^{x_{4}}\left(\varepsilon_{0}\right)^{x_{5}} \omega^{x_{6}}\left(\omega_{c}\right)^{x_{7}}
$$

where the $x$ 's are evaluated by solving the system of homogeneous equations

$$
C x=0
$$

The elements of the coefficient matrix $C$ in (B2) can be obtained by constructing the table [4]

\begin{tabular}{l|rrrrrrr} 
& $x_{1}$ & $x_{2}$ & $x_{3}$ & $x_{4}$ & $x_{5}$ & $x_{6}$ & $x_{7}$ \\
\hline & $H$ & $W$ & $c$ & $\varepsilon$ & $\varepsilon_{0}$ & $\omega$ & $\omega_{c}$ \\
\hline $\mathrm{Kg}$ & 0 & 0 & 0 & -1 & -1 & 0 & 0 \\
$\mathrm{M}$ & 1 & 1 & 1 & -3 & -3 & 0 & 0 \\
$\mathrm{~S}$ & 0 & 0 & -1 & 4 & 4 & -1 & -1 \\
$\mathrm{~A}$ & 0 & 0 & 0 & 2 & 2 & 0 & 0
\end{tabular}

where $\mathrm{Kg}, \mathrm{M}, \mathrm{S}$, and $\mathrm{A}$ are the units of the SI system. Therefore, $\boldsymbol{C}$ is given by

$$
\boldsymbol{C}=\left[\begin{array}{rrrrrrr}
0 & 0 & 0 & -1 & -1 & 0 & 0 \\
1 & 1 & 1 & -3 & -3 & 0 & 0 \\
0 & 0 & -1 & 4 & 4 & -1 & -1 \\
0 & 0 & 0 & 2 & 2 & 0 & 0
\end{array}\right]
$$

The number of independent solutions of (B2) (the same as the number of independent $\pi$-terms) equals the number of elements of $x$ minus the rank of the matrix $\boldsymbol{C}$. In our case the number of elements of $\boldsymbol{x}$ is 7 and the rank of the matrix $\boldsymbol{C}$ is 3 ; hence, we have 4 independent solutions of (B2) 
or $4 \pi$-terms. These independent solutions are given in the table

\begin{tabular}{rrrrrrr}
$x_{1}$ & $x_{2}$ & $x_{3}$ & $x_{4}$ & $x_{5}$ & $x_{6}$ & $x_{7}$ \\
\hline 1 & 0 & -1 & 0 & 0 & 1 & 0 \\
0 & 1 & -1 & 0 & 0 & 1 & 0 \\
0 & 0 & 0 & -1 & 1 & 0 & 0 \\
0 & 0 & 0 & 0 & 0 & -1 & 1 \\
\hline
\end{tabular}

Substituting the value of $x$ 's in (B1) we get the following $\pi$-terms

$$
\begin{array}{cr}
\pi_{1}=\omega H / c, & \pi_{2}=\omega W / c, \\
\pi_{3}=\varepsilon / \varepsilon_{0}=\varepsilon_{r}, & \pi_{4}=\omega_{c} / \omega
\end{array}
$$

From $\pi_{1}$ and $\pi_{2}$ we can get $\pi_{2}^{\prime}=\pi_{2} / \pi_{1}=$ $W / H$. Applying Buckingham's theorem $[4,5]$ the relation between the independent $\pi$-terms can take the form

$$
\pi_{4}=\varphi\left(\pi_{1}, \pi_{2}^{\prime}, \pi_{3}\right)
$$

Therefore,

$$
\omega_{c}=\omega \varphi\left(W / H, \varepsilon_{r}, \omega H / c\right)
$$

But since $\omega_{c}$ is an odd function of $\omega$ (see Section III) we get

$$
\omega_{c}=\omega f\left(W / H, \varepsilon_{r},(\omega H / c)^{2}\right)
$$

\section{ACKNOWLEDGMENT}

The authors thank Dr. J. C. Rautio, President, Sonnet Software, Inc., Liverpool, NY, for making $\boldsymbol{e m}^{\mathrm{TM}}$ available.

\section{REFERENCES}

1. A.H. Zaabab, Q.J. Zhang, and M.S. Nakhala, A neural network modeling approach to circuit opti- mization and statistical design, IEEE Trans Microwave Theory Tech 43 (1995), 1349-1358.

2. P. Watson and K.C. Gupta, EM-ANN models for microstrip vias and interconnects in multilayer circuits, IEEE Trans Microwave Theory Tech 44 (1996), 2495-2503.

3. H. Leung and S. Haykin, Rational function neural network, Neural Computation 5 (1993), 928-938.

4. W.H. Middendorf, Design of devices and systems, Marcel Dekker, New York, 1986, chapter 7.

5. P. Watson, M.Y. Mah, and L.L. Liou, Input variable space reduction using dimensional analysis for artificial neural network modeling, IEEE MTT-S Int Microwave Symp Dig (1999), 269-272.

6. J.W. Bandler, R.M. Biernacki, S.H. Chen, R.H. Hemmers, and K. Madsen, Electromagnetic optimization exploiting aggressive space mapping, IEEE Trans Microwave Theory Tech 43 (1995), 28742882.

7. J.W. Bandler, M.A. Ismail, J.E. Rayas-Sánchez, and Q.J. Zhang, Neuromodeling of microwave circuits exploiting space mapping technology, IEEE Trans Microwave Theory Tech 47 (1999), 928-938.

8. G.C. Temes and J.W. Lapatra, Introduction to circuit synthesis and design, McGraw-Hill, New York, 1977.

9. D.C. Montgomery, Design and analysis of experiments, John Wiley \& Sons, New York, 1991.

10. R. Collin, Foundations for microwave engineering, McGraw-Hill, New York, 1966.

11. $\boldsymbol{e m}^{\mathrm{TM}}$ version $4.0 \mathrm{~b}$, Sonnet Software, Liverpool, NY, 1997.

12. K.C. Gupta, R. Garg, and I.J. Bahl, Microstrip lines and slotlines, Artech House, Dedham, MA, 1979.

13. OSA90/hope ${ }^{\mathrm{TM}}$ version 4.0, Agilent Technologies, Santa Rosa, CA (formerly Optimization Systems Associates, Dundas, Ontario, Canada).

14. M. Kirschning, R. Jansen, and N. Koster, Measurement and computer-aided modeling of microstrip discontinuities by an improved resonator method, IEEE MTT-S Int Microwave Symp Dig (1983), 495-497.

15. D.G. Swanson, Jr., Grounding microstrip lines with via holes, IEEE Trans Microwave Theory Tech 40 (1992), 1719-1721.

\section{BIOGRAPHIES}

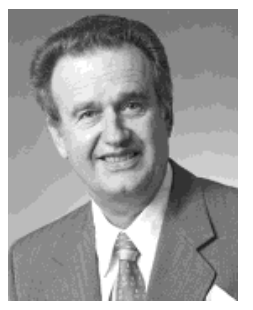

John W. Bandler was born in Jerusalem, on November 9, 1941. He studied at Imperial College of Science and Technology, London, England, from 1960 to 1966. He received the B.Sc. (Eng.), Ph.D. and D.Sc. (Eng.) degrees from the University of London, London, England, in 1963, 1967, and 1976, respectively. He joined Mullard Research Laboratories, Redhill, Surrey, England, in 1966. From 1967 to 1969 he was a Post- doctorate Fellow and Sessional Lecturer at the University of Manitoba, Winnipeg, Canada. Dr. Bandler joined McMaster University, Hamilton, Ontario, Canada, in 1969. He has served as Chairman of the Department of Electrical Engineering and Dean of the Faculty of Engineering. He is currently Professor Emeritus in Electrical and Computer Engineering, directing research in the Simulation Optimization Systems Research Laboratory. $\mathrm{He}$ is a member of the Micronet Network of Centres of Excellence. Dr. Bandler was President of Optimization Systems Associates Inc. (OSA), which he founded in 
1983, until November 20,1997, the date of acquisition of OSA by Hewlett-Packard Company (HP). OSA implemented a first-generation yield-driven microwave CAD capability for Raytheon in 1985, followed by further innovations in linear and nonlinear microwave CAD technology for the Raytheon/Texas Instruments Joint Venture MIMIC Program. OSA introduced the CAE systems RoMPE ${ }^{\mathrm{TM}}$ in 1988,

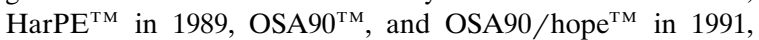
Empipe $^{\mathrm{TM}}$ in 1992, Empipe3D ${ }^{\mathrm{TM}}$ and EmpipeExpress ${ }^{\mathrm{TM}}$ in 1996. OSA created the product empath ${ }^{\mathrm{TM}}$ in 1996 which was marketed by Sonnet Software, Inc., USA. Dr. Bandler is President of Bandler Corporation, which he founded in 1997. Dr. Bandler was an Associate Editor of IEEE Transactions on Microwave Theory and Techniques (1969-1974), and has continued serving as a member of the Editorial Board. He was Guest Editor of the Special Issue of IEEE Transactions on Microwave Theory and Techniques on Computer-Oriented Microwave Practices (1974) and Guest Co-Editor of the Special Issue of the IEEE Transactions on Microwave Theory and Techniques on Process-Oriented Microwave CAD and Modeling (1992). He joined the Editorial Boards of the International Journal of Numerical Modeling in 1987, the International Journal of Microwave and Millimeterwave Computer-Aided Engineering in 1989, and Optimization and Engineering in 1998. He was Guest Editor, International Journal of Microwave and Millimeter-Wave Computer-Aided Engineering. Special Issue on Optimization-Oriented Microwave CAD (1997), and Guest Editor, IEEE Transactions on Microwave Theory and Techniques, Special Issue on Automated Circuit Design Using Electromagnetic Simulators (1997). He is currently Co-Chair of the MTT-1 Technical Committee on Computer-Aided Design. Dr. Bandler has published more than 325 papers from 1965 to 2000. He contributed to Modern Filter Theory and Design. Wiley-Interscience, 1973 and to Analog Methods for Computer-Aided Analysis and Diagnosis, Marcel Dekker, Inc., 1988. Four of his papers have been reprinted in Computer-Aided Filter Design, IEEE Press, 1973, one in each of Microwave Integrated Circuits, Artech House, 1975, Low-Noise Microwave Transistors and Amplifiers, IEEE Press, 1981, Microwave Integrated Circuits, 2nd ed., Artech House, 1985, Statistical Design of Integrated Circuits, IEEE Press, 1987 and Analog Fault Diagnosis, IEEE Press, 1987. Dr. Bandler is a Fellow of the Royal Society of Canada, a Fellow of the Institute of Electrical and Electronics Engineers, a Fellow of the Institution of Electrical Engineers (Great Britain), a Fellow of the Engineering Institute of Canada, a Member of the Association of Professional Engineers of the Province of Ontario (Canada), and a Member of the MIT Electromagnetics Academy. He received the
Automatic Radio Frequency Techniques Group (ARFTG) Automated Measurements Career Award in 1994.

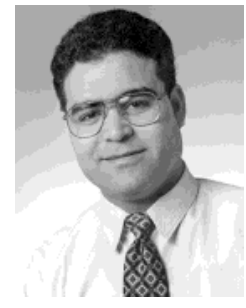

Mostafa A. Ismail was born in Cairo, Egypt, on May 21, 1968. He received the B.Sc. degree in electronics and communications engineering and the Masters degree in engineering mathematics from Cairo University, Egypt, in 1991 and 1995 , respectively, and is currently working toward the Ph.D. degree at McMaster University, Hamilton, Ontario, Canada. In October 1991, he joined the Department of Engineering Mathematics and Physics, Faculty of Engineering, Cairo University. In September 1997, he joined the Department of Electrical and Computer Engineering, McMaster University. His research is carried out in the Simulation Optimization Systems Research Laboratory. His research interests are computer-aided design and modeling of microwave circuits. In 2000, he was granted a one-year Nortel Networks' Ontario Graduate Scholarship in Science and Technology (OGSST).

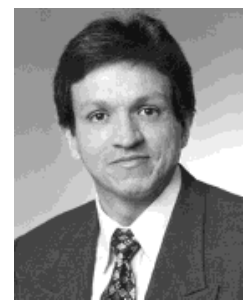

José Ernesto Rayas-Sánchez was born in Guadalajara, Jalisco, Mexico, on December 27,1961 . He received a B.Sc. degree in electronics engineering from the Instituto Technológico y de Estudios Superiores de Occidente (ITESO), Guadalajara, Mexico, in June 1984, and the Masters degree in electrical engineering from the Instituto Technólogico y de Estudios Superiores de Monterrey (ITESM), Monterrey, Mexico, in June 1989, and is currently working toward the Ph.D. degree in electrical engineering at McMaster University, Hamilton, Ontario, Canada. From 1989 to 1997, he was a full-time professor in the Electrical and Computer Engineering Department at ITESO. He joined the Simulation Optimization Systems Research Laboratory, McMaster University, in 1997. His research focuses on the development of novel methods and techniques for computer-aided modeling, design, and optimization of analog wireless electronic circuits and devices exploiting space mapping and artificial neural networks. Mr. Rayas-Sánchez was the recipient of a 1997-2000 Consejo Nacional de Ciencia y Tecnologia (CONACYT) scholarship presented by the Mexican Government, as well as a 2000-2001 Ontario Graduate Scholarship (OGS) presented by the Ministry of Training for Colleges and Universities in Ontario. 